\title{
Erratum to: Barth syndrome without tetralinoleoyl cardiolipin deficiency: a possible ameliorated phenotype
}

\author{
Ann Bowron 1,2,3 • Julie Honeychurch ${ }^{4}$ - Maggie Williams ${ }^{4}$ Beverley Tsai-Goodman ${ }^{2,5}$. \\ Nicol Clayton ${ }^{2}$ - Lucy Jones ${ }^{2}$ - Graham J. Shortland ${ }^{6}$ - Shakeel A. Qureshi ${ }^{7}$. \\ Simon J. R. Heales ${ }^{8,9} \cdot$ Colin G. Steward ${ }^{2,3}$
}

Published online: 15 September 2015

(C) SSIEM 2015

Erratum to: J Inherit Metab Dis (2015) 38:279-286

DOI 10.1007/s10545-014-9747-y

The original version of this article unfortunately contained a mistake. The reference by Whited is incomplete. The corrected reference is:

Whited K, Baile MG, Currier P, Claypool SM (2013) Seven functional classes of Barth syndrome mutation. Human Molecular Genetics 22:483-492.

The online version of the original article can be found at http://dx.doi.org/ 10.1007/s10545-014-9747-y.

Ann Bowron
ann.bowron@bristol.ac.uk

Department of Clinical Biochemistry, University Hospitals Bristol NHS Trust, Bristol BS2 8HW, UK

2 NHS Barth Syndrome Service, Bristol Royal Hospital for Children, University Hospitals Bristol NHS Trust, Bristol BS2 8BJ, UK

3 School of Cellular \& Molecular Medicine, School of Medical Sciences, University Walk, Bristol BS8 1TD, UK

4 Bristol Genetics Laboratory, North Bristol NHS Trust, Bristol BS10 $5 \mathrm{NB}, \mathrm{UK}$
5 Department of Paediatric Cardiology, Bristol Royal Hospital for Children, University Hospitals Bristol NHS Trust, Bristol BS2 8BJ, UK

6 Department of Metabolic Disease, University Hospitals Wales, Cardiff CF14 4XW, UK

7 Department of Paediatric Cardiology, Evelina Children's Hospital, Guy's and St Thomas' NHS Foundation Trust, London SE1 7EH, UK

8 Department of Chemical Pathology, Great Ormond Street Hospital NHS Foundation Trust, London WC1N 3JH, UK

9 University College London Institute of Child Health, London WC1N 1EH, UK 